\title{
An inhomogeneous Jarník theorem
}

\author{
Yann Bugeaud (Strasbourg)
}

\begin{abstract}
We compute the generalized Hausdorff dimension of sets of points in $\mathbf{R}^{s}$ which satisfy an inhomogeneous system of Diophantine inequalities infinitely often. This provides an inhomogeneous analogue of a classical result of Jarnik.
\end{abstract}

\section{Introduction}

A classical result of Khintchine [20] asserts that, given a non-increasing function $\Psi$ : $\mathbf{Z}_{>0} \rightarrow \mathbf{R}_{>0}$, the set

$\mathcal{K}(\Psi):=\{\xi \in \mathbf{R}:|q \xi-p|<\Psi(q)$ for infinitely many integers $p$ and $q$ with $q \geq 1$.

has full Lebesgue measure if the sum $\sum_{q \geq 1} \Psi(q)$ diverges, while it has Lebesgue measure zero otherwise. However, since the Hausdorff measure allows us to discriminate between null sets, we may search for a more precise statement in the convergence case. For instance, Jarník [18] and Besicovitch [7] proved, independently, that for any $\lambda>1$ the Hausdorff dimension of $\mathcal{K}\left(x \mapsto x^{-\lambda}\right)$ is equal to $2 /(\lambda+1)$. In fact, Jarník's result is much more precise, since it involves generalized Hausdorff measures (for the definition, see Section 2). It also deals with simultaneous Diophantine approximation: for an integer $s \geq 1$ and $\Psi$ as above, Jarník considers the set

$$
\begin{aligned}
\mathcal{K}(\Psi):=\left\{\underline{\xi}=\left(\xi_{1}, \ldots, \xi_{s}\right)\right. & \in \mathbf{R}^{s}: \max _{1 \leq u \leq s}\left|q \xi_{u}-p_{u}\right|<\Psi(q) \\
& \text { for infinitely many } \left.\left(p_{1}, \ldots, p_{s}, q\right) \in \mathbf{Z}^{s+1} \text { with } q \geq 1\right\}
\end{aligned}
$$

and he proved the following statement ([7], Sätze 3 und 4).

Theorem J. Let $\Psi$ as above, with $\lim _{x \rightarrow \infty} \Psi(x)=0$. Let $s \geq 1$ be integer. Let $f: \mathbf{R}_{\geq 0} \rightarrow$ $\mathbf{R}_{\geq 0}$ be a strictly increasing continuous function such that $f(0)=0$ and $f(x) / x^{s}$ tends monotonically to infinity when $x$ tends to 0 . Assume that the sum $\sum_{x=1}^{+\infty} \Psi^{s}(x)$ converges

1991 Mathematics Subject Classification : 11J83, 11J20. 
and that the functions $x \mapsto x^{s+1} f(2 \Psi(x) / x)$ and $x \mapsto x \Psi^{s}(x)$ are non-increasing. Then $\mathcal{H}^{f}(\mathcal{K}(\Psi))=+\infty$ if the sum

$$
\sum_{x=1}^{+\infty} x^{s} f(2 \Psi(x) / x)
$$

diverges, and $\mathcal{H}^{f}(\mathcal{K}(\Psi))=0$ otherwise.

Theorem $\mathrm{J}$ may be regarded as a definitive result, and a natural question is whether the same conclusion also holds for any set

$$
\begin{aligned}
\mathcal{K}_{\underline{\alpha}}(\Psi):=\left\{\underline{\xi}=\left(\xi_{1}, \ldots, \xi_{s}\right) \in \mathbf{R}^{s}: \max _{1 \leq u \leq s}\left|q \xi_{u}-p_{u}-\alpha_{u}\right|<\Psi(q)\right. \\
\\
\text { for infinitely many } \left.\left(p_{1}, \ldots, p_{s}, q\right) \in \mathbf{Z}^{s+1} \text { with } q \geq 1\right\} .
\end{aligned}
$$

where $\underline{\alpha}=\left(\alpha_{1}, \ldots, \alpha_{s}\right)$ is some fixed real $s$-tuple. The answer seems not to follow from Theorem J. In fact, inhomogeneous Diophantine approximation is rather different (and can be much more difficult) than the homogeneous case, and often it deserves different methods. For instance, an inhomogeneous analogue of Dirichlet's theorem does not hold. However, Schmidt [25] managed to prove the $s$-dimensional analogue of the above-mentioned result of Khintchine, namely that the set $\mathcal{K}_{\underline{\alpha}}(\Psi)$ has full Lebesgue measure if the sum $\sum_{q>1} \Psi^{s}(q)$ diverges, and $\mathcal{K}_{\underline{\alpha}}(\Psi)$ is a null set otherwise. In the latter case, Levesley [21] has recently shown (as a special case of a more general result) that the Hausdorff dimension of $\mathcal{K}_{\underline{\alpha}}(\Psi)$ is equal to $(s+1) /(\lambda+1)$, where $\lambda=\liminf _{x \rightarrow \infty}(-\log \Psi(x)) / \log x$ is supposed to be $>1 / s$. We like to mention that the so-called doubly metric case, where one considers the joint measure of the set of points $(\underline{\xi}, \underline{\alpha})$ in $\mathbf{R}^{s} \times \mathbf{R}^{s}$ is easier. We refer to Dickinson [12] and Dodson [15] for a general form of the inhomogeneous version of the doubly metric Jarník-Besicovitch theorem.

One of the purposes of the present work is to strengthen Levesley's result by proving the inhomogeneous analogue of Theorem J. To this end, we define the notion of optimal regular system, which asks more than the concept of regular system, introduced by Baker \& Schmidt [1] in order to compute the Hausdorff dimension of sets of real numbers very close to infinitely many algebraic numbers of bounded degree. We prove (see Theorem 3) a general statement valid for approximation of real numbers by points belonging to an optimal regular system and we prove that the set of points which occurs for determinating the measure of $\mathcal{K}_{\underline{\alpha}}$ is indeed an optimal regular system (see Theorem 2). At the end of Section 2, we point out that our Theorem 3 can be applied to a wide class of Diophantine approximation problems.

\section{Statement of our results}

Before stating our results, we briefly recall the definition of the generalized Hausdorff measure of a set $E \subset \mathbf{R}^{s}$, where $s \geq 1$ is an integer. We denote by $\operatorname{diam} \mathcal{U}=\sup \left\{\|x-y\|_{\infty}\right.$ : $x, y \in \mathcal{U}\}$ the diameter of a bounded set $\mathcal{U} \subset \mathbf{R}^{s}$, i.e. the greatest distance apart of any pair of points in $\mathcal{U}$. Let $f: \mathbf{R}_{>0} \rightarrow \mathbf{R}_{>0}$ be a dimension function, i.e. a positive valued 
function, increasing in a neighbourhood of the origin and such that $\lim _{x \rightarrow 0} f(x)=0$. Let $\delta$ be a positive real number and set

$$
h(f, \delta, E):=\inf \sum_{j} f\left(\operatorname{diam} \mathcal{U}_{j}\right),
$$

where the infimum is taken over all finite or enumerable families of sets $\mathcal{U}_{j} \subset \mathbf{R}^{s}$ of diameter $\operatorname{diam} \mathcal{U}_{j}<\delta$ which cover $E$. As $\delta$ decreases to 0 , the quantity $h(f, \delta, E)$ increases to a finite or infinite limit $\mathcal{H}^{f}(E)$, called the Hausdorff $f$-measure of $E$. When there exists some positive real number $t$, such that $f(x)=x^{t}$, we simply write $\mathcal{H}^{t}$ instead of $\mathcal{H}^{f}$ : this defines the $t$-dimensional Hausdorff measure. Then, the Hausdorff dimension of $E$, which we denote by $\operatorname{dim} E$, is the real number $t_{0} \in[0,1]$ defined by

$$
t_{0}=\inf \left\{t: \mathcal{H}^{t}(E)=0\right\}=\sup \left\{t: \mathcal{H}^{t}(E)=+\infty\right\}
$$

We refer the reader to e.g. the books of Bernik \& Dodson [6], of Falconer [17] and of Rogers [22] for complementary definitions and results on Hausdorff measures.

Our main result states as follows.

Theorem 1. Let $s \geq 1$ and $\underline{\alpha}=\left(\alpha_{1}, \ldots, \alpha_{s}\right)$ be a real $s$-tuple. Let $\Psi$ be a non-increasing function, with $\lim _{x \rightarrow \infty} \Psi(x)=0$. Let $f: \mathbf{R}_{>0} \rightarrow \mathbf{R}_{>0}$ be a dimension function such that $f(x) / x^{s}$ tends monotonically to infinity as $x$ tends to 0 . Assume that the sum $\sum_{x=1}^{+\infty} \Psi^{s}(x)$ converges and that the functions $x \mapsto x^{s+1} f(2 \Psi(x) / x)$ and $x \mapsto x \Psi^{s}(x)$ are non-increasing. Then $\mathcal{H}^{f}\left(\mathcal{K}_{\underline{\alpha}}(\Psi)\right)=+\infty$ if the sum

$$
\sum_{x=1}^{+\infty} x^{s} f(2 \Psi(x) / x)
$$

diverges, and $\mathcal{H}^{f}\left(\mathcal{K}_{\underline{\alpha}}(\Psi)\right)=0$ otherwise.

Remark: The technical hypotheses are the same as those in Theorem J, and are not very restrictive. Recall that, according to Schmidt's result quoted in the Introduction, the set $\mathcal{K}_{\underline{\alpha}}(\Psi)$ has full Lebesgue measure if the sum $\sum_{x=1}^{+\infty} \Psi^{s}(x)$ diverges.

Theorem 1 gives the generalized Hausdorff measure $\mathcal{H}^{f}$ of $\mathcal{K}_{\underline{\alpha}}(\Psi)$ and so is more discrimating than the Hausdorff dimension result given by Levesley's theorem quoted above. We take the following example from [14], to which we refer the reader for complementary observations. Let $\underline{\alpha}$ be a given $s$-tuple of real numbers. Let $\tau>(s+1) / s$, and, for any $\varepsilon \geq 0$, define the function $\Psi_{\varepsilon}$ by

$$
\Psi_{\varepsilon}(x)=x^{-\tau}(\log x)^{-\tau(1+\varepsilon) /(s+1)}, \quad \text { for } x \geq 3 .
$$

It follows from Levesley's result that

$$
d:=\operatorname{dim}\left(\mathcal{K}_{\underline{\alpha}}\left(\Psi_{\varepsilon}\right)\right)=\frac{s+1}{\tau}
$$


thus the Hausdorff dimension of $\mathcal{K}_{\underline{\alpha}}\left(\Psi_{\varepsilon}\right)$ does not depend on $\varepsilon$. For $0<\varepsilon_{1}<\varepsilon_{2}$, we consider the dimension function $f$ defined by

$$
f(x):=x^{d}\left(\log x^{-1 / \tau}\right)^{\varepsilon_{1}} .
$$

An easy calculation shows that Theorem 1 yields that

$$
\mathcal{H}^{f}\left(\mathcal{K}_{\underline{\alpha}}\left(\Psi_{\varepsilon_{1}}\right)\right)=+\infty
$$

whilst

$$
\mathcal{H}^{f}\left(\mathcal{K}_{\underline{\alpha}}\left(\Psi_{\varepsilon_{2}}\right)\right)=0 .
$$

Thus, the (generalized) Hausdorff measure $\mathcal{H}^{f}$ distinguishes between the sizes of the sets $\mathcal{K}_{\underline{\alpha}}\left(\Psi_{\varepsilon}\right)$, unlike the $t$-dimensional Hausdorff measure.

In [5], Beresnevich, Dickinson \& Velani have applied the main result of [14] to determine the precise (generalized) Hausdorff dimension of sets of matrices with "exact $t$ logarithmic order" (i.e. the approximating functions $\Psi$ are of the shape $\Psi(q)$ equals $q^{d}$ times a finite product of powers of iterated logarithms). See also [8] for a similar discussion in the context of approximation by real algebraic numbers of bounded degree. With the same arguments as in [5], we may deduce an inhomogeneous analogue of Theorems 1 and 2 of [5], in the particular case $m=1$.

The strategy for proving Theorem 1 proceeds in two steps, and rests on the notion of regular system of points in $\mathbf{R}$, introduced by Baker \& Schmidt [1] for determinating the Hausdorff dimension of sets of real numbers close to infinitely many algebraic numbers of bounded degree. However, when we search for sharper results, involving generalized Hausdorff measure, we need to refine this notion, by introducing that of optimal regular system.

Definition 1. Let $E \subset \mathbf{R}$ be a, bounded or unbounded, interval. Let $\mathcal{S}=\left(\alpha_{n}\right)_{n \geq 1}$ be a sequence of real numbers in $E$. Then $\mathcal{S}$ is called an optimal regular system of points in $E$ if there exist positive constants $c_{1}, c_{2}$ and $c_{3}$, depending only on $\mathcal{S}$, and, for any bounded interval $I \subset E$, a number $k_{0}=k_{0}(\mathcal{S}, I)$ such that, for any $K \geq k_{0}$, there exist integers

$$
c_{1} K \leq i_{1}<\ldots<i_{t} \leq K
$$

with $\alpha_{i_{j}} \in I$ for any $1 \leq j \leq t$,

$$
\left|\alpha_{i_{j}}-\alpha_{i_{\ell}}\right| \geq \frac{c_{2}}{K} \quad(1 \leq j \neq \ell \leq t)
$$

and

$$
c_{3}|I| K \leq t \leq|I| K .
$$

For instance, it follows from Dirichlet's theorem that the set of rational numbers $p / q$ ordered firstly by increasing values of the maximum of $|p|$ and $|q|$, then by increasing numerical order, is an optimal regular system. Further, Beresnevich [2] proved that, for 
any integer $n \geq 2$, the set of real algebraic numbers of degree $n$, with a suitable ordering, is an optimal regular system (he did not show the existence of $c_{1}$ in (1), but this easily follows from his proof, see Proposition 1 of [8]). The same holds for the set of algebraic integers of degree $n$, see [9] (here also, the positive constant $c_{1}$ in (1) is not explicitely given; however, a suitable value follows from inequality (8) of [9]).

We refer the reader to the survey of Beresnevich, Bernik \& Dodson [4] for more explanations and examples on regular systems and optimal regular systems (which they call best possible regular systems). In the present work, we have chosen a slightly different presentation of this notion, since we have imposed an ordering for the set of points forming our optimal regular system (Rynne [23] did the same for a regular system). This allows us to establish a general result which, as will be explained below, may be applied to quite a few different problems.

The importance of the notion of optimal regular system appears firstly in the work of Beresnevich [2], where he considers the particular case of the set of algebraic numbers of fixed degree. However, using his arguments, it is easy to get the following result, proved in [3] (see also Lemma 3 in [4]).

Theorem B. Let $E \subset \mathbf{R}$ be a, bounded or unbounded, interval. Let $\mathcal{S}=\left(\alpha_{n}\right)_{n \geq 1}$ be a sequence of real numbers which is an optimal regular system in $E$. Let $\Psi: \mathbf{Z}_{>0} \rightarrow \mathbf{R}_{>0}$ be a non-increasing function and set

$$
E\left(\alpha_{j}\right):=\left\{\xi \in E:\left|\xi-\alpha_{j}\right|<\Psi(j)\right\}
$$

for any $j \geq 1$ and

$$
E(\Psi)=\limsup _{j \rightarrow+\infty} E\left(\alpha_{j}\right) .
$$

Then the set $E(\Psi)$ is a null set if the sum $\sum_{j \geq 1} \Psi(j)$ converges and $E(\Psi)$ has full measure if this sum diverges.

A natural extension of Theorem $B$ is the computation of the generalized Hausdorff measure of $E(\Psi)$ in the converging case, which was the purpose of [8] with $\mathcal{S}$ being the set of algebraic numbers of fixed degree. A general statement follows from Theorem 3 below.

As Beresnevich [3], we may extend the notion of (optimal) regular system to sequences of points in $\mathbf{R}^{s}$, with $s \geq 2$. In the present paper, we use the following notation. The cube $\mathcal{C}=\mathcal{C}(\underline{\alpha}, V)$ in $\mathbf{R}^{s}$ is the compact subset of $\mathbf{R}^{s}$, centered at the point $\underline{\alpha}$ and having volume $V$. In other words, writing $\underline{\alpha}=\left(\alpha^{(1)}, \ldots, \alpha^{(s)}\right)$, we have

$$
\mathcal{C}(\underline{\alpha}, V)=\prod_{1 \leq u \leq s}\left[\alpha^{(u)}-V^{1 / s} / 2, \alpha^{(u)}+V^{1 / s} / 2\right] .
$$

We denote by $\operatorname{Vol}(\mathcal{C})$ the volume of a cube $\mathcal{C}$ and by $\langle\mathcal{C}\rangle$ its sidelength, hence we have $\operatorname{Vol}(\mathcal{C})=\langle\mathcal{C}\rangle^{s}$

Definition 2. Let $s \geq 2$ be an integer. Let $E \subset \mathbf{R}^{s}$ be a Cartesian product of closed intervals. Let $\mathcal{S}=\left(\underline{\alpha}_{n}\right)_{n \geq 1}$, with $\underline{\alpha}_{n}=\left(\alpha_{n}^{(1)}, \ldots, \alpha_{n}^{(s)}\right)$ be a sequence of points in $E$. Then $\mathcal{S}$ is called an optimal regular system of points in $E$ if there exist positive constants $c_{1}, c_{2}$ 
and $c_{3}$, depending only on $\mathcal{S}$, and, for any cube $\mathcal{C} \subset E$, a number $k_{0}=k_{0}(\mathcal{S}, \mathcal{C})$ such that, for any $K \geq k_{0}$, there exist integers

$$
c_{1} K \leq i_{1}<\ldots<i_{t} \leq K
$$

with $\underline{\alpha}_{i_{j}} \in \mathcal{C}$ for any $1 \leq j \leq t$

$$
\max _{1 \leq u \leq s}\left|\alpha_{i_{j}}^{(u)}-\alpha_{i_{\ell}}^{(u)}\right| \geq \frac{c_{2}}{K^{1 / s}} \quad(1 \leq j \neq \ell \leq t)
$$

and

$$
c_{3} \operatorname{Vol}(\mathcal{C}) K \leq t \leq \operatorname{Vol}(\mathcal{C}) K .
$$

Remark: We may replace the cubes occurring in Definition 2 by Cartesian products of compact intervals.

In all what follows, we denote by $\{x\}$ the integral part of a real number $x$. In order to prove Theorem 1 , we first have to prove that, given a real $s$-tuple $\underline{\alpha}=\left(\alpha_{1}, \ldots, \alpha_{s}\right)$, the set of points

$$
\left(\left\{\frac{p_{1}+\alpha_{1}}{q}\right\}, \ldots,\left\{\frac{p_{s}+\alpha_{s}}{q}\right\}\right), \quad q \geq 1, p_{1}, \ldots, p_{s} \geq 0
$$

with a suitable ordering, form an optimal regular system. When $\alpha_{1}=\ldots=\alpha_{s}=0$, this follows from Dirichlet's theorem, however, in the general case, this is slightly more complicated.

Theorem 2. Let $\underline{\alpha}=\left(\alpha_{1}, \ldots, \alpha_{s}\right)$ be a fixed real s-tuple. The set $\mathcal{S}_{\underline{\alpha}}$ composed of the points

$$
\left(\left\{\frac{p_{1}+\alpha_{1}}{q}\right\}, \ldots,\left\{\frac{p_{s}+\alpha_{s}}{q}\right\}\right)
$$

with

$$
p_{1}, \ldots, p_{s}, q \in \mathbf{Z}, q \geq 1, \quad \text { and } \quad 0 \leq p_{1}, \ldots, p_{s} \leq q-1
$$

ordered firstly by increasing values of $q$, then by increasing values of $\max _{1 \leq u \leq s} p_{u}$, is an optimal regular system in $[0,1]^{s}$.

The proof of Theorem 2 heavily depends on an approach due to Khintchine [19]. We do not give full details: we merely point out how to use an intermediate result of [19] to get our desired statement.

Remark: Theorem 2 can be compared with Lemma 4 of Levesley [21]. This lemma is sufficiently sharp to imply that $\mathcal{S}_{\underline{\alpha}}$ is a regular system, and to get (using classical tools) the exact Hausdorff dimension of sets $\mathcal{K}_{\underline{\alpha}}(\Psi)$. However, because of the term $\log ^{\sigma} N$, we cannot infer from it that $\mathcal{S}_{\underline{\alpha}}$ is an optimal regular system.

As previously mentioned, the generalization of Theorem $\mathrm{J}$ for set of real numbers close to infinitely many real algebraic numbers of given degree $n$ has been obtained in [8]. The idea of the proof originates in [18] and was also used by Dickinson \& Velani [14] in a quite similar context. We constructed inductively a Cantor set and a positive measure on it, and 
we applied Frostmann's lemma (see Proposition 2 in Section 4). It appears that the only property of the set of real algebraic numbers of degree $n$ we used is the fact that, with a suitable ordering, it forms an optimal regular system.

Theorem 3. Let $s \geq 1$. Let $E \subset \mathbf{R}^{s}$ be a Cartesian product of intervals. Let $\mathcal{S}=\left(\underline{\alpha}_{n}\right)_{n \geq 1}$ be a sequence of points $\underline{\alpha}_{n}=\left(\alpha_{n}^{(1)}, \ldots, \alpha_{n}^{(s)}\right)$ of real numbers, which is an optimal regular system in $E$. Let $\Psi: \mathbf{Z}_{>0} \rightarrow \mathbf{R}_{>0}$ be a non-increasing function and set

$$
E\left(\underline{\alpha}_{j}\right):=\left\{\underline{\xi}=\left(\xi^{(1)}, \ldots, \xi^{(s)}\right) \in E: \max _{1 \leq u \leq s}\left|\xi^{(u)}-\alpha_{j}^{(u)}\right|<\Psi(j)\right\}
$$

for any $j \geq 1$ and

$$
E(\Psi)=\limsup _{j \rightarrow+\infty} E\left(\underline{\alpha}_{j}\right)
$$

Let $f$ be a dimension function such that $\lim _{x \rightarrow 0} f(x) / x^{s}=+\infty$ and $x \mapsto f(x) / x^{s}$ is nonincreasing in a neighbourhood of the origin. Assume that the sum $\sum_{j \geq 1} \Psi^{s}(j)$ converges and that $x \mapsto x(f(2 \Psi(x)))$ is non-increasing and tends to 0 when $x$ goes to infinity. Then we have

$$
\mathcal{H}^{f}(E(\Psi))=+\infty \quad \text { if } \quad \sum_{j \geq 1} f(2 \Psi(j)) \quad \text { diverges }
$$

and

$$
\mathcal{H}^{f}(E(\Psi))=0 \quad \text { if } \quad \sum_{j \geq 1} f(2 \Psi(j)) \quad \text { converges. }
$$

Remark: The assumption on the convergence of the sum $\sum_{j \geq 1} \Psi^{s}(j)$ cannot be removed. Indeed, when this sum diverges, then the set $E(\Psi)$ has full Lebesgue measure, by a result of Beresnevich [3], extending Theorem B above.

We keep the notation of Theorem 3 and we let $\Psi_{1}, \ldots \Psi_{s}: \mathbf{Z}_{>0} \rightarrow \mathbf{R}_{>0}$ be given non-increasing functions. A possible generalization consists in studying the sets

$$
E\left(\underline{\alpha}_{j}\right):=\left\{\underline{\xi}=\left(\xi^{(1)}, \ldots, \xi^{(s)}\right) \in E: \max _{1 \leq u \leq s}\left|\xi^{(u)}-\alpha_{j}^{(u)}\right|<\Psi_{u}(j)\right\}
$$

for any $j \geq 1$ and

$$
E\left(\Psi_{1}, \ldots, \Psi_{s}\right)=\limsup _{j \rightarrow+\infty} E\left(\underline{\alpha}_{j}\right) .
$$

When $\mathcal{S}$ is the optimal regular system given by Theorem 2 with $\alpha_{1}=\ldots=\alpha_{s}=0$, Rynne [24] has determined the Hausdorff dimension of the sets $E\left(\Psi_{1}, \ldots, \Psi_{s}\right)$. In a subsequent work, we plan to give an analogue of Theorem 1 for these sets.

Applying Theorem 3 with the optimal regular system formed by the real algebraic numbers of degree $n$, we recover the main result of [8], without an extra technical hypothesis. See the remark following Proposition 1 for more details.

Some examples of regular systems and optimal regular systems have been stated above (see also [4]), but our list is not exhaustive. We briefly mention, without any details, two other examples, as well as another important application of Theorem 3. 
In a recent paper, Dickinson \& Dodson [13] have calculated the Hausdorff dimension of points close to the circle $\mathbf{S}^{1}$. To this end, they have used ubiquity, but, as they mention, they could also have used the notion of regular system. Through their proof, they have indeed shown (see Lemma 2 of [13]) that a certain set of points form an optimal regular system. Thus, thanks to Theorem 3 , it is possible to compute the generalized Hausdorff measure of the set

$$
\left\{(x, y) \in \mathbf{S}^{1}: \max \{\|q x\|,\|q y\|\}<\Psi(q) \text { for infinitely many integers } q \geq 1\right\}
$$

where $\Psi$ is a non-increasing function such that $\lim _{x \rightarrow+\infty} x \Psi(x)=0$, and $\|\cdot\|$ denotes the distance to the nearest integer.

In [11], we have computed the Hausdorff measure of sets of real numbers close to infinitely many elements of the sequence $(\{n \alpha\})_{n \geq 1}$, where $\alpha$ is a given irrational number. Following our proof, it turns out that this sequence is an optimal regular system when $\alpha$ has bounded partial quotients, hence we may apply Theorem 3 in this case in order to get a sharper statement.

As explained in [10] the notion of intersective sets, first introduced by Falconer [16], is well adapted in the context of Diophantine approximation. Indeed, following the approach of [10], it turns out that Proposition 1 below is enough to assert that we end up with an intersective set. We refer the reader to [10] for more details on this notion.

\section{Proof of Theorem 2}

Let $s \geq 1$ be an integer and let $\underline{\alpha}=\left(\alpha_{1}, \ldots, \alpha_{s}\right)$ be a fixed real $s$-tuple. Let $\mathcal{C}$ be a cube in $[0,1]^{s}$ with sidelength $\langle\mathcal{C}\rangle$. Let $Q \geq 10$ be real. For any $s$-tuple $\xi=\left(\xi_{1}, \ldots, \xi_{s}\right)$ in $[0,1]^{s}$, let $q(\underline{\xi})$ denote the smallest positive integer such that there exist integers $p_{1}, \ldots, p_{s}$ with

$$
\left|q(\underline{\xi}) \xi_{u}-p_{u}\right|<\frac{1}{Q}, \quad 1 \leq u \leq s .
$$

It follows from Dirichlet's theorem that $q(\underline{\xi}) \leq Q^{s}$. Let $\mathcal{C}_{0}$ denote the set of $\underline{\xi} \in[0,1]^{s}$ for which we have $q(\underline{\xi}) \leq 5^{-s} Q^{s}$, and set $\mathcal{C}_{1}=\mathcal{C} \backslash \mathcal{C}_{0}$. The Lebesgue measure $\bar{\lambda}\left(\mathcal{C}_{0}\right)$ of $\mathcal{C}_{0}$ is bounded by

$$
\sum_{x=1}^{\left[5^{-s} Q^{s}\right]+1}\left(\frac{2}{x Q}\right)^{s}(\langle\mathcal{C}\rangle x+2)^{s}
$$

hence we have

$$
\lambda\left(\mathcal{C}_{0}\right) \leq \frac{\langle\mathcal{C}\rangle^{s}}{2}
$$

and

$$
\lambda(\mathcal{C}) \leq 2 \lambda\left(\mathcal{C}_{1}\right)
$$

if $Q$ is large enough (in terms of $s$ and of $\langle\mathcal{C}\rangle$ ). 
We now follow the argument of Khintchine [19]. Let $\xi \in \mathcal{C}_{1}$. We point out that our $5^{-s}$ (resp. our $\left.Q\right)$ corresponds to the $\gamma\left(\right.$ resp. the $\left.t^{\prime}\right)$ of [19]. Hence, by [19, p. 415], there exist a constant $\kappa_{1}$, depending only on $s$, and integers $x, y^{(1)}, \ldots, y^{(s)}$ such that

$$
\left|x \xi_{u}-y^{(u)}-\alpha_{u}\right|<\frac{\kappa_{1}}{q(\underline{\xi})^{1 / s}}, \quad 1 \leq u \leq s
$$

and

$$
q(\underline{\xi}) \leq x<2 q(\underline{\xi}) .
$$

In fact, in [19], the inequalities (4) are replaced by $0 \leq x<q(\underline{\xi})$. However, since $x$ is determined modulo $q(\underline{\xi})$, the conclusion of Khintchine also holds with the condition (4).

Since $5^{-s} Q^{s}<q(\underline{\xi}) \leq Q^{s}$, it follows from (3) and (4) that, for $1 \leq u \leq s$, we have

$$
\left|\xi_{u}-\frac{y^{(u)}+\alpha_{u}}{x}\right|<\frac{\kappa_{1}}{x q(\underline{\xi})^{1 / s}} \leq \frac{25 \kappa_{1}}{Q^{s+1}}
$$

and

$$
5^{-s} Q^{s} \leq x \leq 2 Q^{s}
$$

Let

$$
\underline{\gamma}_{i_{1}}=\left(\frac{y_{i_{1}}^{(1)}+\alpha_{1}}{x_{i_{1}}}, \ldots, \frac{y_{i_{1}}^{(s)}+\alpha_{s}}{x_{i_{1}}}\right), \ldots, \underline{\gamma}_{i_{t}}=\left(\frac{y_{i_{t}}^{(1)}+\alpha_{1}}{x_{i_{t}}}, \ldots, \frac{y_{i_{t}}^{(s)}+\alpha_{s}}{x_{i_{t}}}\right)
$$

be a maximal subset of distinct elements of $\mathcal{S}_{\underline{\alpha}}$ with

$$
5^{-s} Q^{s} \leq x_{i_{h}} \leq 2 Q^{s}
$$

and

$$
\max _{1 \leq u \leq s}\left|\underline{\gamma}_{i_{h}}^{(u)}-\underline{\gamma}_{i_{j}}^{(u)}\right| \geq 1 / Q^{s+1}, \quad \text { for } \quad 1 \leq h \neq j \leq t .
$$

Our assumption of maximality implies that each $\underline{\gamma}=\left(\left(y^{(1)}+\alpha_{1}\right) / x, \ldots,\left(y^{(s)}+\alpha_{s}\right) / x\right)$ in $\mathcal{S}_{\alpha}$ with $5^{-s} Q^{s} \leq x \leq 2 Q^{s}$ is at most at a distance $1 / Q^{s+1}$ from some $\underline{\gamma}_{i_{\ell}}$. Hence, by (5) and (6), if $\underline{\xi} \in \mathcal{C}_{1}$, then there exists $1 \leq \ell \leq t$ such that for any $1 \leq u \leq s$ we have

$$
\left|\xi_{u}-\gamma_{i_{\ell}}^{(u)}\right| \leq \frac{25 \kappa_{1}+1}{Q^{s+1}}
$$

Thus, the cubes centered at the points $\underline{\gamma}_{i_{h}}, 1 \leq h \leq t$, and with sidelength $2\left(25 \kappa_{1}+1\right) / Q^{s+1}$ cover the set $\mathcal{C}_{1}$, whence, by (2),

$$
\operatorname{Vol}(\mathcal{C})=\lambda(\mathcal{C}) \leq 2 \lambda\left(\mathcal{C}_{1}\right) \leq 2 t\left(\frac{2\left(25 \kappa_{1}+1\right)}{Q^{s+1}}\right)^{s}
$$


and there exists a positive constant $\kappa_{2}$ depending only on $s$ such that

$$
t \geq \kappa_{2} \operatorname{Vol}(\mathcal{C}) Q^{s(s+1)} .
$$

Our ordering of $\mathcal{S}_{\underline{\alpha}}$ implies that, for any integer $m \geq 1$, the elements of $\mathcal{S}_{\underline{\alpha}}$ with denominator equal to $\bar{m}$ are exactly the $\underline{\gamma}_{j}^{\prime}$ 's with $1+2^{s}+\ldots+(m-1)^{s}+1 \leq j \leq$ $1+2^{s}+\ldots+m^{s}$. Hence, by $(6)$, there exists a constant $\kappa_{3}$, depending only on $s$, such that

$$
\kappa_{3} Q^{s(s+1)} \leq i_{1}, \ldots, i_{t} \leq 2^{s+1} Q^{s(s+1)} .
$$

Setting $K=2^{s+1} Q^{s(s+1)}$, we infer from (9), (7) and (8) that $\mathcal{S}_{\underline{\alpha}}$ is an optimal regular system in $[0,1]^{s}$.

\section{Proofs of Theorems 1 and 3}

We first deal with the easy half of Theorem 3 . We assume that the sum $\sum_{j \geq 1} f(2 \Psi(j))$ converges, and we apply the Hausdorff-Cantelli Lemma (see e.g. [6], Lemma 3.9). More precisely, we observe that, for any integer $\ell \geq 1$, the union of the cubes centered at the points $\underline{\alpha}_{j}, j \geq \ell$, and with sidelength $2 \Psi(j)$ covers the set $E(\Psi)$. Keeping the notation of Section 2, for any $\delta>0$ and any integer $\ell$ sufficiently large in terms of $\delta$, we infer from the monotonicity of the function $x \mapsto x(f(2 \Psi(x))$ ) (which we invoke since the diameter of a cube is not equal to its sidelength) that there exists an absolute constant $\kappa$ such that

$$
h(f, \delta, E(\Psi)) \leq \kappa \sum_{j \geq \ell} f(2 \Psi(j)) .
$$

Hence, $h(f, \delta, E(\Psi))=0$ since the above sum converges. As $\delta$ is arbitrarily small, we deduce that

$$
\mathcal{H}^{f}(E(\Psi))=0
$$

as claimed.

Before proceeding with the remaining part of the proof of Theorem 3, we state a useful auxiliary result.

Proposition 1. Let $\mathcal{S}=\left(\underline{\alpha}_{n}\right)_{n \geq 1}$ be an optimal regular system in a Cartesian product of intervals $E$ in $\mathbf{R}^{s}$. Let $\mathcal{C}$ be a cube in $E$. Let $F$ be a positive, non-increasing function such that the sum $\sum_{j \geq 1} F(j)$ diverges and $x \mapsto x F(x)$ is non-increasing and tends to 0 as $x$ goes to infinity. Let $m>0$. Then there exist a positive constant $c=c(\mathcal{S}) \leq 1$, depending only on $\mathcal{S}$, and integers $m \leq i_{1}<\ldots<i_{t}$ such that the cubes

$$
\mathcal{C}\left(\underline{\alpha}_{i_{j}}, F\left(i_{j}\right)\right)
$$

are included in $J$ and pairwise disjoint, and

$$
\sum_{j=1}^{t} F\left(i_{j}\right) \geq c(\mathcal{S}) \operatorname{Vol}(\mathcal{C})
$$


Remark: When $\mathcal{S}$ is the set of real algebraic numbers of degree $n$, Proposition 1 has been proved in [8], but with an extra technical hypothesis. To remove this assumption, the key point is to define the variable $H$ in terms of the constant $c_{1}$ (with the notation occurring in the proof below).

Proof : Since $\mathcal{S}$ is an optimal regular system, a consequence of Definition 2 is the existence of positive constants $c_{1}, c_{2}, c_{3}$ and $k_{0}$ such that, for any $K \geq k_{0}$, there are integers

$$
c_{1} K \leq i_{1}<\ldots<i_{t} \leq K
$$

with $\underline{\alpha}_{i_{j}} \in \mathcal{C}$ for any $1 \leq j \leq t$

$$
\begin{gathered}
\max _{1 \leq u \leq s}\left|\alpha_{i_{j}}^{(u)}-\alpha_{i_{\ell}}^{(u)}\right| \geq \frac{c_{2}}{K^{1 / s}} \quad(1 \leq j \neq \ell \leq t), \\
\mathcal{C}\left(\underline{\alpha}_{i_{j}}, F\left(i_{j}\right)\right) \subset \mathcal{C}
\end{gathered}
$$

and

$$
c_{3} \operatorname{Vol}(\mathcal{C}) K \leq t \leq \operatorname{Vol}(\mathcal{C}) K .
$$

We have to justify (10). Notice first that, if $K$ is sufficiently large, we have $F\left(i_{j}\right)<c_{2}^{s} / K$ since $F$ is non-increasing and $\lim _{x \rightarrow \infty} x F(x)=0$. At most $2 s\left(\operatorname{Vol}(\mathcal{C}) c_{2}^{-1} K^{1 / s}\right)^{s-1}$ among the cubes $\mathcal{C}\left(\underline{\alpha}_{i_{j}}, F\left(i_{j}\right)\right)$ are not completely included in $\mathcal{C}$, hence, taking a smaller positive value for $c_{3}$ if necessary, we get that (10) holds for all cubes.

Decreasing the value of $c_{1}$ if necessary, we may assume that $H:=2 c_{1}^{-1}$ is an integer with $H \geq 16^{s}$. By assumption, the integral $\int_{1}^{\infty} F(t) d t$ diverges, whence, by the change of variables $t=c_{1} H^{u}$, the integral

$$
\int_{1}^{\infty} H^{u} F\left(c_{1} H^{u}\right) d u
$$

also diverges. Since $x \mapsto x F(x)$ is non-increasing, it follows that

$$
\sum_{k \geq 1} H^{k} F\left(c_{1} H^{k}\right)=+\infty
$$

Let $k_{1} \geq k_{0}$ be an integer such that

$$
\begin{gathered}
x F(x) \leq 8^{-s-7} c_{1} c_{2}^{s} \quad \text { for any } x \geq c_{1} H^{k_{1}} \\
c_{1} H^{k_{1}}>m
\end{gathered}
$$

and

$$
c_{3} \operatorname{Vol}(\mathcal{C}) H^{k_{1}} \geq 10 .
$$

By (11) and (12), there exists an integer $M$ with

$$
\frac{c_{2}^{s}}{8^{s+4}} \leq \sum_{k=k_{1}}^{k_{1}+M} H^{k} F\left(c_{1} H^{k}\right) \leq \frac{c_{2}^{s}}{8^{s+3}}
$$


We first apply the definition of optimal regular system with the integer $K=H^{k_{1}}$. By (13), we get a set $\mathcal{A}(0)$ of $\left[\operatorname{Vol}(\mathcal{C}) c_{3} H^{k_{1}}\right]$ integers $\geq m$ such that the cubes

$$
\mathcal{C}\left(\underline{\alpha}_{j}, F(j)\right), \quad j \in \mathcal{A}(0),
$$

are contained in $\mathcal{C}$. These are pairwise disjoint. Indeed, on the one hand, we infer from (12) that

$$
8^{s+7} F\left(c_{1} H^{k_{1}}\right)<c_{2}^{s} H^{-k_{1}}
$$

hence

$$
\left\langle\mathcal{C}\left(\underline{\alpha}_{j}, F(j)\right)\right\rangle<2^{-3} c_{2} H^{-k_{1} / s} \quad \text { for } j \in \mathcal{A}(0) .
$$

On the other hand, we have

$$
\max _{1 \leq u \leq s}\left|\underline{\alpha}_{h}^{(u)}-\underline{\alpha}_{j}^{(u)}\right| \geq c_{2} H^{-k_{1} / s} \quad \text { for any } h, j \text { in } \mathcal{A}(0)
$$

which, together with (26), allows us to conclude that the cubes are pairwise disjoint.

We now repeat this process with $K=H^{k_{1}+\ell}$ for $1 \leq \ell \leq M$. Since we want to end up with disjoint cubes, we have to remove some $\underline{\alpha}_{j}$ 's at each step. To do this, we proceed by induction, assuming that, for some $0 \leq \ell \leq M-1$, we have already construct disjoint sets of integers $\mathcal{A}(0), \ldots, \mathcal{A}(\ell)$ such that the cubes

$$
\mathcal{C}\left(\underline{\alpha}_{j}, F(j)\right), \quad j \in \mathcal{A}(0) \cup \ldots \cup \mathcal{A}(\ell),
$$

are pairwise disjoint.

Applying the definition of optimal regular system with the integer $K=H^{k_{1}+\ell+1}$, there is a set $\tilde{\mathcal{A}}(\ell+1)$ of $\left[c_{3} \operatorname{Vol}(\mathcal{C}) H^{k_{1}+\ell+1}\right]$ integers with

$$
\max _{1 \leq u \leq s}\left|\underline{\alpha}_{h}^{(u)}-\underline{\alpha}_{j}^{(u)}\right| \geq c_{2} H^{-\left(k_{1}+\ell+1\right)} \quad \text { for any } h, j \text { in } \tilde{\mathcal{A}}(\ell+1) .
$$

and such that the cubes

$$
\mathcal{C}\left(\underline{\alpha}_{j}, F(j)\right), \quad j \in \tilde{\mathcal{A}}(\ell+1),
$$

are contained in $\mathcal{C}$ and pairwise disjoint. Recall that at each step $h$, with $0 \leq h \leq \ell$, we have constructed not more than $\left[c_{3} \operatorname{Vol}(\mathcal{C}) H^{k_{1}+h}\right]$ cubes of volume at most equal to $F\left(c_{1} H^{k_{1}+h}\right)$. Hence, to be sure that the new cubes have empty intersection with the previous constructed cubes, we have, by using (17) and the trivial upper bound $(a+b)^{s} \leq 2^{s}\left(a^{s}+b^{s}\right)$, valid for any positive numbers $a$ and $b$, to remove at most

$$
\begin{aligned}
N_{\ell} & :=\sum_{h=0}^{\ell} c_{3} \operatorname{Vol}(\mathcal{C}) H^{k_{1}+h}\left(F\left(c_{1} H^{k_{1}+h}\right)^{1 / s}+2 c_{2} H^{-\left(k_{1}+\ell+1\right) / s}\right)^{s} H^{k_{1}+\ell+1} c_{2}^{-s} \\
& =\sum_{h=0}^{\ell} 2^{s} c_{3} \operatorname{Vol}(\mathcal{C}) H^{k_{1}+h}\left(F\left(c_{1} H^{k_{1}+h}\right) H^{k_{1}+\ell+1} c_{2}^{-s}+2^{s}\right)
\end{aligned}
$$


points $\underline{\alpha}_{j}$ with $j \in \tilde{\mathcal{A}}(\ell+1)$.

By (15) and $\ell \leq M$, we have

$$
\begin{aligned}
N_{\ell} & \leq 2^{s} c_{3} \operatorname{Vol}(\mathcal{C}) H^{k_{1}+\ell+1}\left(\sum_{k=k_{1}}^{k_{1}+M} c_{2}^{-s} H^{k} F\left(c_{1} H^{k}\right)+2^{s} \sum_{k \geq 1} H^{-k}\right) \\
& \leq c_{3} \operatorname{Vol}(\mathcal{C}) H^{k_{1}+\ell+1} / 2
\end{aligned}
$$

since $H \geq 16^{s}$, by assumption.

Thus, there remain at least $\left[c_{3} \operatorname{Vol}(\mathcal{C}) H^{k_{1}+\ell+1} / 2\right]$ cubes, each of them having volume at least equal to $F\left(H^{k_{1}+\ell+1}\right)$. Since $H=2 c_{1}^{-1}$, the total volume of all the cubes constructed is then, by (12) and (15), at least (we use (14) to ensure that $\left[c_{3} \operatorname{Vol}(\mathcal{C}) H^{k_{1}+\ell+1} / 2\right]$ is greater than $\left.c_{3} \operatorname{Vol}(\mathcal{C}) H^{k_{1}+\ell+1} / 3\right)$

$$
\begin{aligned}
\frac{1}{3} \sum_{h=0}^{M} \operatorname{Vol}(\mathcal{C}) c_{3} H^{k_{1}+h} F\left(H^{k_{1}+h}\right) & \geq \frac{1}{6} \sum_{h=0}^{M} \operatorname{Vol}(\mathcal{C}) c_{3} c_{1} H^{k_{1}+h+1} F\left(c_{1} H^{k_{1}+h+1}\right) \\
& \geq \frac{c_{1} c_{3}}{6} \operatorname{Vol}(\mathcal{C})\left(\frac{c_{2}^{s}}{8^{s+4}}-H^{k_{1}} F\left(c_{1} H^{k_{1}}\right)\right) \\
& \geq \frac{c_{1}^{2} c_{3}}{8} \operatorname{Vol}(\mathcal{C})\left(\frac{c_{2}^{s}}{8^{s+4}}-\frac{c_{2}^{s}}{8^{s+5}}\right) \\
& \geq \frac{c_{1} c_{2}^{s} c_{3}}{8^{s+5}} \operatorname{Vol}(\mathcal{C}) .
\end{aligned}
$$

This implies that

$$
\sum_{j \in \mathcal{A}(0) \cup \ldots \cup \mathcal{A}(M)} F(j) \geq \frac{c_{1} c_{2}^{s} c_{3}}{8^{s+5}} \operatorname{Vol}(\mathcal{C}),
$$

as expected.

\section{Proof of Theorem 3:}

Thanks to the above proposition, we are now able to prove Theorem 3. Our approach originates in the work of Jarník. We construct inductively finite sets of compact cubes: at each step $\ell$ we associate to any cube a finite number of sub-cubes of controlled volume and not too close to each other. The intersection of all these cubes is our Cantor set $\mathbf{K}$, on which we define a probability measure $\mu$. We conclude by applying the mass distribution principle (Proposition 2 below). This method has also been used by Dickinson \& Velani [14], and is very clearly explained in the book of Falconer [17, Chapter 4].

- Construction of the Cantor set $\mathbf{K}$ contained in $\mathbf{R}^{s}$.

It follows from the assumptions of Theorem 3 that the function $x \mapsto f(2 \Psi(x))$ is nonincreasing. Denote by $c=c(\mathcal{S})$ the constant given by Proposition 1 applied to the optimal regular system $\mathcal{S}$. Since the Hausdorff measure $\mathcal{H}^{f}$ is subadditive, there is no restriction in assuming that the set $E$ is included in $[0,1]^{s}$. 
Let $\lambda>0$ be a real number. We construct the set $\mathbf{K}(1)$ by applying Proposition 1 to the cube $\mathcal{C}=\mathbf{K}(0)=[0,1]^{s}$, the function

$$
F: x \mapsto \lambda c \operatorname{Vol}(\mathbf{K}(0)) f(2 \Psi(x))
$$

and an integer $m_{0} \geq 2$ such that

$$
F(x)=\lambda c \operatorname{Vol}(\mathbf{K}(0)) f(2 \Psi(x))>4^{s} \Psi^{s}(x) \quad \text { for all } x \geq m_{0}
$$

Such $m_{0}$ does exist since

$$
\lim _{u \rightarrow+\infty} \Psi(u)=0 \quad \text { and } \quad \lim _{x \rightarrow 0} \frac{f(x)}{x^{s}}=+\infty
$$

Hence, we get a set $\mathcal{A}(1):=\left\{i_{1}^{(1)}, \ldots, i_{t_{1}}^{(1)}\right\}$ of distinct integers greater than $m_{0}$, such that the cubes

$$
I(1,0, i):=\mathcal{C}\left(\underline{\alpha}_{i}, F(i)\right), \quad i \in \mathcal{A}(1),
$$

are pairwise disjoint. By (18), for any $i \in \mathcal{A}(1)$, the cube

$$
\mathbf{K}(1,0, i)=\mathbf{K}(1, i):=\mathcal{C}\left(\underline{\alpha}_{i}, 2^{s} \Psi^{s}(i)\right)
$$

is contained in $I(1,0, i)$. We set

$$
\mathbf{K}(1)=\bigcup_{i \in \mathcal{A}(1)} \mathbf{K}(1,0, i)=\bigcup_{i \in \mathcal{A}(1)} \mathbf{K}(1, i)
$$

and we stress that the union is disjoint. Further, Proposition 1 asserts that the function $F$ satisfies

$$
\sum_{i \in \mathcal{A}(1)} F(i) \geq c \operatorname{Vol}(\mathbf{K}(0))
$$

which yields that

$$
\begin{aligned}
\sum_{i \in \mathcal{A}(1)} f(\langle\mathbf{K}(1,0, i)\rangle) & =\sum_{i \in \mathcal{A}(1)} f(2 \Psi(i)) \\
& =\frac{1}{\lambda c \operatorname{Vol}(\mathbf{K}(0))} \sum_{i \in \mathcal{A}(1)} F(i) \geq \frac{1}{\lambda}
\end{aligned}
$$

Assume now that for some $\ell \geq 1$, we have constructed a set of integers $\mathcal{A}(\ell):=$ $\left\{i_{1}^{(\ell)}, \ldots, i_{t_{\ell}}^{(\ell)}\right\}$ and a set $\mathbf{K}(\ell)$, which is the union of $t_{\ell}$ compact, disjoint cubes $\mathbf{K}(\ell, j)$, $j \in \mathcal{A}(\ell)$, centered at the points $\underline{\alpha}_{j}$ and with volume

$$
\operatorname{Vol}(\mathbf{K}(\ell, j))=\langle\mathbf{K}(\ell, j)\rangle^{s}=2^{s} \Psi^{s}(j) .
$$


Then, we construct the set $\mathbf{K}(\ell+1)$ as the disjoint union of compact sub-cubes of the $\mathbf{K}(\ell, j)$ 's. To this end, for any integer $j$ with $1 \leq j \leq t_{\ell}$, we apply Proposition 1 to $\mathbf{K}(\ell, j)$, with the function

$$
F_{\ell, j}: x \rightarrow c f(2 \Psi(x)) \frac{\operatorname{Vol}(\mathbf{K}(\ell, j))}{f(\langle\mathbf{K}(\ell, j)\rangle)}
$$

and with a real number $m_{\ell, j}$ such that

$$
F_{\ell, j}(x)>4^{s} \Psi^{s}(x) \text { for any } x \geq m_{\ell, j} .
$$

Thus, we get a set $\mathcal{A}(\ell+1, j)$ of integers $i_{h}^{(\ell+1, j)}$, with $1 \leq h \leq t_{\ell, j}$, all greater than $m_{\ell, j}$, such that the cubes

$$
\mathcal{C}\left(\underline{\alpha}_{i}, F_{\ell, j}(i)\right), \quad i \in \mathcal{A}(\ell+1, j),
$$

are pairwise disjoint. Hence, by (20), the cubes

$$
\mathbf{K}(\ell+1, j, i):=\mathcal{C}\left(\underline{\alpha}_{i}, 2^{s} \Psi^{s}(i)\right), \quad i \in \mathcal{A}(\ell+1, j),
$$

are, a fortiori, pairwise disjoint. For each $1 \leq j \leq t_{\ell}$, we have built $t_{\ell, j}$ cubes $\mathbf{K}(\ell+1, j, i)$ contained in the cube $\mathbf{K}(\ell, j)$. Thus, we have constructed

$$
t_{\ell+1}:=\sum_{j=1}^{t_{\ell}} t_{\ell, j}
$$

cubes contained in $\mathbf{K}(\ell)$, which we reindex as

$$
\mathbf{K}(\ell+1,1), \ldots, \mathbf{K}\left(\ell+1, t_{\ell+1}\right) .
$$

We set

$$
\mathbf{K}(\ell+1)=\bigcup_{j=1}^{t_{\ell+1}} \mathbf{K}(\ell+1, j),
$$

which is the union of $t_{\ell+1}$ pairwise disjoint cubes. To complete the inductive process, it remains for us to set

$$
\mathcal{A}(\ell+1):=\bigcup_{j=1}^{t_{\ell}} \mathcal{A}(\ell+1, j) .
$$

It is easy to check that the union is disjoint.

Since Proposition 1 yields, for any $1 \leq j \leq t_{\ell}$, that

$$
\sum_{i \in \mathcal{A}(\ell+1, j)} F_{\ell, j}(i) \geq c \operatorname{Vol}(\mathbf{K}(\ell, j)),
$$

we get

$$
\begin{aligned}
\sum_{i \in \mathcal{A}(\ell+1, j)} f(\langle\mathbf{K}(\ell+1, j, i)\rangle) & =\frac{1}{c} \sum_{i \in \mathcal{A}(\ell+1, j)} \frac{f(\langle\mathbf{K}(\ell, j)\rangle)}{\operatorname{Vol}(\mathbf{K}(\ell, j))} F_{\ell, j}(i) \\
& \geq \frac{1}{c} \frac{f(\langle\mathbf{K}(\ell, j)\rangle)}{\operatorname{Vol}(\mathbf{K}(\ell, j))} c \operatorname{Vol}(\mathbf{K}(\ell, j))=f(\langle\mathbf{K}(\ell, j)\rangle) .
\end{aligned}
$$

The following important lemma easily follows from (19) and (21). We recall that, for any $\ell \geq 1$, the set $\mathbf{K}(\ell)$ is the union of exactly $t_{\ell}$ disjoint cubes. 
Lemma 1. For any $\ell \geq 1$ and any $1 \leq j \leq t_{\ell}$, we have

$$
\sum_{i \in \mathcal{A}(\ell+1, j)} f(\langle\mathbf{K}(\ell+1, j, i)\rangle) \geq f(\langle\mathbf{K}(\ell, j)\rangle) .
$$

Further, for any $\ell \geq 1$, we have

$$
\sum_{\mathcal{C} \in \mathbf{K}(\ell)} f(\langle\mathcal{C}\rangle) \geq \sum_{\mathcal{C} \in \mathbf{K}(1)} f(\langle\mathcal{C}\rangle) \geq \frac{1}{\lambda}
$$

Proof : The first assertion is a rewriting of (21). An easy induction based on (22) and (19) allows us to get the second part of the lemma.

Then, we define the Cantor set

$$
\mathbf{K}:=\bigcap_{\ell=0}^{\infty} \mathbf{K}(\ell)
$$

Clearly, we have

$$
\mathbf{K} \subset E(\Psi) .
$$

Further, $\mathbf{K}(1)$ depends on the real number $\lambda$, given at the beginning of the process. Thus, $\mathbf{K}$ also depends on $\lambda$. In order to simplify the notation, we do not use an additional index in order to point out this dependence.

- Construction of a probability measure with support in $\mathbf{K}$.

In order to apply the mass distribution principle (Proposition 2 below), we construct a probability measure $\mu$ with support in $\mathbf{K}$ such that for any cube $\mathcal{C}$ of sidelength $\langle\mathcal{C}\rangle$ we have

$$
\mu(\mathcal{C}) \leq \frac{4^{s} \lambda}{c} f(\langle\mathcal{C}\rangle)
$$

We define inductively the measure $\mu$ on each cube of $\mathbf{K}(\ell)$ as follows. We set $\mu(\mathbf{K}(0))=$ 1. Let $\ell \geq 0$ and let $\mathcal{C}$ be one of the $t_{\ell+1}$ cubes building $\mathbf{K}(\ell+1)$. With the previous notation, there exists a unique pair $\left(j_{0}, i_{0}\right)$ with $1 \leq j_{0} \leq t_{\ell}$ and $1 \leq i_{0} \leq t_{\ell, j_{0}}$ such that $\mathcal{C}=\mathbf{K}\left(\ell+1, j_{0}, i_{0}\right)$. Then, we set

$$
\mu(\mathcal{C}):=\frac{f(\langle\mathcal{C}\rangle)}{\sum_{i \in \mathcal{A}\left(\ell, j_{0}\right)} f\left(\left\langle\mathbf{K}\left(\ell+1, j_{0}, i\right)\right\rangle\right)} \mu\left(\mathbf{K}\left(\ell, j_{0}\right)\right)
$$

The measure of each cube arising in the construction of $\mathbf{K}$ is well defined. Further, this measure extends to each of the Borelian sets of $\mathbf{K}(0)$ (see e.g. [22], Proposition 1.7). 
Fact. The measure $\mu$ defined as above has support in $\mathbf{K}$ and, for any Borelian set $F$ contained in $\mathbf{K}(0)$, it satisfies

$$
\mu(F)=\inf _{\mathcal{C} \in \mathcal{J}} \mu(\mathcal{C}),
$$

where the infimum is taken over all the coverings $\mathcal{J}$ of $F$ by cubes $\mathcal{C}$ belonging to the union of the $\mathbf{K}(\ell), \ell \geq 0$.

Lemma 2. For any $\ell \geq 1$ and any cube $\mathcal{C}$ belonging to $\mathbf{K}(\ell)$, we have

$$
\mu(\mathcal{C}) \leq \lambda f(\langle\mathcal{C}\rangle)
$$

In particular, the cube $\mathcal{C}$ satisfies (24).

Proof : Let $\ell \geq 1,1 \leq j \leq t_{\ell}$ and $i \in \mathcal{A}(\ell, j)$. The cubes $\mathbf{K}(\ell, j)$ and $\mathbf{K}(\ell+1, j, i)$ belong, respectively, to the sets $\mathbf{K}(\ell)$ and $\mathbf{K}(\ell+1)$, and they satisfy $\mathbf{K}(\ell+1, j, i) \subset \mathbf{K}(\ell, j)$. The definition (25) of $\mu$ and (22) imply that

$$
\frac{\mu(\mathbf{K}(\ell+1, j, i))}{f(\langle\mathbf{K}(\ell+1, j, i)\rangle)} \leq \frac{\mu(\mathbf{K}(\ell, j))}{f(\langle\mathbf{K}(\ell, j)\rangle)} .
$$

Since $\mu(\mathbf{K}(0))=1$ and, by Lemma $1, \sum_{\mathcal{C} \in \mathbf{K}(1)} f(\langle\mathcal{C}\rangle) \geq 1 / \lambda$, the definition of $\mu$ implies $\mu(\mathcal{C}) \leq \lambda f(\langle\mathcal{C}\rangle)$ for any cube $\mathcal{C}$ belonging to $\mathbf{K}(1)$. Combined with (26), this proves Lemma 2 , for $c \leq 1$.

Now, we have to prove $(24)$ for an arbitrary cube $\mathcal{C}$. Let $\delta>0$ be sufficiently small in order that any cube of sidelength $\delta$ intersects at most one component of $\mathbf{K}(1)$.

Lemma 3. If $\mathcal{C}$ is a cube included in $\mathbf{K}(0)$ of volume less than $\delta$, then

$$
\mu(\mathcal{C}) \leq \frac{4^{s} \lambda}{c} f(\langle\mathcal{C}\rangle)
$$

Proof : We may assume that $\mathcal{C}$ intersects $\mathbf{K}$, since otherwise we get $\mu(\mathcal{C})=0$ and $(24)$ is trivially satisfied. Further, if $\mathcal{C}$ intersects, for any $\ell$, one and only one component of $\mathbf{K}(\ell)$, then $\mu(\mathcal{C})=0$ since the volume of the components of $\mathbf{K}(\ell)$ tends to 0 when $\ell$ tends to infinity: (24) is also trivially satisfied. It only remains for us to consider the case where there exists an integer $\ell$ such that $\mathcal{C}$ intersects exactly one cube from $\mathbf{K}(\ell)$, which, according to the preceeding notation, we denote by $\mathbf{K}(\ell, j)$, and at least two cubes from $\mathbf{K}(\ell+1)$, which we denote by $\mathbf{K}\left(\ell+1, j, i_{1}\right), \ldots, \mathbf{K}\left(\ell+1, j, i_{a}\right)$, with $a \geq 2$. We assume that, for $1 \leq h \leq a$, the cube $\mathbf{K}\left(\ell+1, j, i_{h}\right)$ is centered at the vector of index $i_{h}^{(\ell+1, j)}$ and has volume $2^{s} \Psi^{s}\left(i_{h}^{(\ell+1, j)}\right)$. Our choice of $\delta$ ensures that $\ell \geq 1$. For convenience, we omit the superscript $(\ell+1, j)$ in the sequel.

We may assume that $\alpha_{i_{1}}<\ldots<\alpha_{i_{a}}$. We denote by $F_{\ell, j}$ the function used above to construct the compact sub-cubes of $\mathbf{K}(\ell, j)$. We recall that the cubes $\mathcal{C}\left(\alpha_{i_{h}}, F_{\ell, j}\left(i_{h}\right)\right)$, $1 \leq h \leq a$, are pairwise disjoint and that $\mathcal{C}$ intersects all the cubes $\mathbf{K}\left(\ell+1, j, i_{h}\right), 1 \leq h \leq$ 
$a$, which have volume $2^{s} \Psi^{s}\left(i_{h}\right)$. Then, for any $1 \leq h \leq a$, the volume of the intersection $\mathcal{C}\left(\alpha_{i_{h}}, F_{\ell, j}\left(i_{h}\right)\right) \cap \mathbf{K}\left(\ell+1, j, i_{h}\right)$ is not less than

$$
2^{-s}\left(\left\langle\mathcal{C}\left(\alpha_{i_{h}}, F_{\ell, j}\left(i_{h}\right)\right)\right\rangle-\left\langle\mathbf{K}\left(\ell+1, j, i_{h}\right)\right\rangle\right)^{s}=2^{-s}\left(F_{\ell, j}\left(i_{h}\right)^{1 / s}-2 \Psi\left(i_{h}\right)\right)^{s} \geq 2^{-2 s} F_{\ell, j}\left(i_{h}\right),
$$

by $(20)$. Hence, we have

$$
\operatorname{Vol}(\mathcal{C} \cap \mathbf{K}(\ell, j)) \geq \sum_{h=1}^{a} 2^{-2 s} F_{\ell, j}\left(i_{h}\right)
$$

and, using the definition of $F_{\ell, j}$, it follows that

$$
\begin{aligned}
\operatorname{Vol}(\mathcal{C} \cap \mathbf{K}(\ell, j)) & \geq \frac{1}{4^{s}} \sum_{h=1}^{a} F_{\ell, j}\left(i_{h}\right) \\
& \geq \frac{c}{4^{s}} \frac{\operatorname{Vol}(\mathbf{K}(\ell, j))}{f(\langle\mathbf{K}(\ell, j)\rangle)} \sum_{h=1}^{a} f\left(2 \Psi\left(i_{h}\right)\right) \\
& \geq \frac{c}{4^{s}} \frac{\operatorname{Vol}(\mathbf{K}(\ell, j))}{f(\langle\mathbf{K}(\ell, j)\rangle)} \sum_{h=1}^{a} f\left(\left\langle\mathbf{K}\left(\ell+1, j, i_{h}\right)\right\rangle\right) .
\end{aligned}
$$

Lemma 2 and (27) imply that

$$
\begin{aligned}
\mu(\mathcal{C})=\mu(\mathcal{C} \cap \mathbf{K}(\ell, j)) & \leq \sum_{h=1}^{a} \mu\left(\mathbf{K}\left(\ell+1, j, i_{h}\right)\right) \\
& \leq \lambda \sum_{h=1}^{a} f\left(\left\langle\mathbf{K}\left(\ell+1, j, i_{h}\right)\right\rangle\right) \\
& \leq \frac{4^{s} \lambda}{c} \frac{f(\langle\mathbf{K}(\ell, j)\rangle)}{\operatorname{Vol}(\mathbf{K}(\ell, j))} \operatorname{Vol}(\mathcal{C} \cap \mathbf{K}(\ell, j)) \\
& \leq \frac{4^{s} \lambda}{c} f(\langle\mathcal{C} \cap \mathbf{K}(\ell, j)\rangle),
\end{aligned}
$$

since the function $x \mapsto f(x) / x^{s}$ is non-increasing on a neighbourhood of 0 and $\operatorname{Vol}(\mathcal{C} \cap$ $\mathbf{K}(\ell, j)) \leq \operatorname{Vol}(\mathcal{C})$. As the function $f$ increases, we get $(24)$, as claimed.

- Conclusion.

Proposition 2. Let $m$ be a probability measure with support in a bounded set $E \subset \mathbf{R}^{s}$. Assume that there exist $\lambda>0$ and $\delta>0$ such that

$$
m(\mathcal{U}) \leq \lambda f(\operatorname{diam} \mathcal{U})
$$

for any set $\mathcal{U}$ with $\operatorname{diam} \mathcal{U} \leq \delta$. Then, we have

$$
\mathcal{H}^{f}(E) \geq \frac{1}{\lambda}
$$


Proof : We give a proof, although it can be found in [14] and in [17, page 55]. Let $\rho \leq \delta$ be positive. If $\left\{\mathcal{U}_{i}\right\}$ denotes a finite or enumerable covering of $E$ such that the diameter of any set $\mathcal{U}_{i}$ is at most $\rho$, then we have

$$
1=m(E)=m\left(\bigcup_{i} \mathcal{U}_{i}\right) \leq \sum_{i} m\left(\mathcal{U}_{i}\right) \leq \lambda \sum_{i} f\left(\operatorname{diam} \mathcal{U}_{i}\right)
$$

Therefore, we have $h(f, \rho, E) \geq \lambda^{-1}$ for any $\rho \leq \delta$. The lemma follows by letting $\delta$ tend to 0 .

The measure $\mu$ constructed above is a probability measure with support in $\mathbf{K}$. By Lemma 3 , it satisfies the hypotheses of the mass distribution principle. Indeed, if $\mathcal{U}$ is a subset of $[0,1]^{s}$, there exists a constant $\kappa \geq 1$, depending only on $s$, such that $\mathcal{U}$ is contained in a cube of sidelength at most $\kappa \operatorname{diam} \mathcal{U}$. By Lemma 3, if diam $\mathcal{U}$ is small enough, we get that

$$
\mu(\mathcal{U}) \leq \frac{4^{s} \lambda}{c} f(\kappa \operatorname{diam} \mathcal{U}) \leq \frac{4^{s} \lambda \kappa}{c} f(\operatorname{diam} \mathcal{U})
$$

since, by assumption, the function $x \mapsto f(x) / x$ is non-increasing in a neighbourhood of the origin. Thus, Proposition 2 yields that

$$
\mathcal{H}^{f}(\mathbf{K}) \geq \frac{c}{4^{s} \lambda \kappa}
$$

whence, by (23),

$$
\mathcal{H}^{f}(E(\Psi)) \geq \frac{c}{4^{s} \lambda \kappa}
$$

Since $\lambda>0$ can be chosen arbitrarily small, we obtain

$$
\mathcal{H}^{f}(E(\Psi))=+\infty
$$

as asserted.

\section{Proof of Theorem 1:}

It is enough to compute the $\mathcal{H}^{f}$ measure of the set $\mathcal{K}_{\underline{\alpha}}(\Psi) \cap[0,1]^{s}$. To this end, we apply Theorem 3 with the optimal regular system $\mathcal{S}_{\underline{\alpha}}$ (which is optimal, by Theorem 2) and the function $\tilde{\Psi}$ defined, for any positive integer $m$, by

$$
\tilde{\Psi}\left(1^{s}+\ldots+(m-1)^{s}+1\right)=\ldots=\tilde{\Psi}\left(1^{s}+\ldots+(m-1)^{s}+m^{s}\right)=\frac{\Psi(m)}{m} .
$$

Thus, we have $\mathcal{K}_{\alpha}(\Psi)=E(\tilde{\Psi})$. Further, we observe that, for any integer $N \geq 1$, we have

$$
\sum_{q=1}^{N} q f(2 \Psi(q) / q)=\sum_{j=1}^{1^{s}+\ldots+N^{s}} f(2 \tilde{\Psi}(j))
$$


which shows that the sum

$$
\sum_{j \geq 1} f(2 \tilde{\Psi}(j))
$$

diverges if, and only if, the sum

$$
\left.\sum_{q \geq 1} q f(2 \Psi(q) / q)\right)
$$

diverges. Hence, Theorem 3 yields Theorem 1.

\section{References}

[1] A. Baker and W. M. Schmidt, Diophantine approximation and Hausdorff dimension, Proc. London Math. Soc. 21 (1970), 1-11.

[2] V. Beresnevich, On approximation of real numbers by real algebraic numbers, Acta Arith. 90 (1999), 97-112.

[3] V. Beresnevich, Application of the concept of regular system of points in metric number theory, Vesti NAN Belarusi. Phys-Mat. Ser. (2000), no. 1.

[4] V. Beresnevich, V. I. Bernik and M. M. Dodson, Regular systems, ubiquity and Diophantine approximation, Proceedings of the conference dedicated to the 60th birthday of A. Baker, Birkhäuser Verlag. To appear.

[5] V. Beresnevich, H. Dickinson and S. L. Velani, Sets of exact "logarithmic order" in the theory of Diophantine approximation, Math. Annalen 321 (2001), 253-273.

[6] V. I. Bernik and M. M. Dodson, Metric Diophantine approximation on manifolds, Cambridge Tracts in Mathematics 137, Cambridge University Press, 1999.

[7] A. S. Besicovitch, Sets of fractional dimension (IV): on rational approximation to real numbers, J. London Math. Soc. 9 (1934), 126-131.

[8] Y. Bugeaud, Approximation par des nombres algébriques de degré borné et dimension de Hausdorff, J. Number Theory. To appear.

[9] Y. Bugeaud, Approximation by algebraic integers and Hausdorff dimension, J. London Math. Soc. To appear.

[10] Y. Bugeaud, Ensembles intersectifs et approximation diophantienne. Submitted.

[11] Y. Bugeaud, A note on inhomogeneous Diophantine approximation. Submitted.

[12] H. Dickinson, A remark on the Jarnik-Besicovitch theorem, Glasgow Math. J. 39 (1997), 233-236.

[13] H. Dickinson and M. M. Dodson, Diophantine approximation and Hausdorff dimension on the circle, Math. Proc. Cambridge Phil. Soc. 130 (2001), 515-522.

[14] H. Dickinson and S. L. Velani, Hausdorff measure and linear forms, J. reine angew. Math. 490 (1997), 1-36. 
[15] M. M. Dodson, A note on metric inhomogeneous Diophantine approximation, J. Austral. Math. Soc. (Series A) 62 (1997), 175-185.

[16] K. Falconer, Classes of sets with large intersections, Mathematika 32 (1985), 191-205.

[17] K. Falconer, Fractal Geometry : Mathematical Foundations and Applications, John Wiley \& sons, 1990.

[18] V. Jarník, Über die simultanen Diophantische Approximationen, Math. Z. 33 (1931), $505-543$.

[19] A. Khintchine, Ein Satz über lineare diophantische Approximationen, Math. Ann. 112 (1936), 398-415.

[20] A. Khintchine, Continued Fractions, University of Chicago Press, 1964.

[21] J. Levesley, A general inhomogeneous Jarnik-Besicovitch theorem, J. Number Theory 71 (1998), 65-80.

[22] C. A. Rogers, Hausdorff Measures, Cambridge University Press, Cambridge, 1970.

[23] B. P. Rynne, Regular and ubiquitous systems and $\mathcal{M}_{\infty}^{s}$-dense sequences, Mathematika 39 (1992), 234-243.

[24] B. P. Rynne, Hausdorff dimension and generalised simultaneous Diophantine approximation, Bull. London Math. Soc. 30 (1998), 365-376.

[25] W. M. Schmidt, Metrical theorems on fractional parts of sequences, Trans. Amer. Math. Soc. 110 (1964), 493-518.

Yann Bugeaud

Université Louis Pasteur

U. F. R. de mathématiques

7, rue René Descartes

67084 STRASBOURG Cedex

e-mail : bugeaud@math.u-strasbg.fr 